\title{
ON THE COVERING DIMENSION OF SUBSPACES OF PRODUCT OF SORGENFREY LINES ${ }^{1}$
}

\author{
ALI A. FORA
}

\begin{abstract}
Let $S$ denote the Sorgenfrey line. Then the following results are proved in this paper:

(i) If $X$ is a nonempty subspace of $S^{\boldsymbol{\mu}_{0}}$, then $\operatorname{dim} X=0$.

(ii) For any nonempty separable space $X \subset S^{\mu_{0}}, \operatorname{dim} X^{m}=0$ for any cardinal $\boldsymbol{m}$.
\end{abstract}

1. Introduction. The question of whether $\operatorname{dim}(X \times Y) \leqslant \operatorname{dim} X+\operatorname{dim} Y$ for topological spaces $X$ and $Y$ has long been considered (see e.g., [G, pp. 263 and 277]). By $\operatorname{dim} X$, or the covering dimension of $X$, we mean the least integer, $n$, such that each finite cozero cover of $X$ has a finite cozero refinement of order $n$. (A cover is of order $n$ if and only if each point of the space is contained in at most $n+1$ elements of the cover. All spaces considered are completely regular.)

Researchers have long worked on the above problem, and only recently Wage [W] and Przymusiński [P] constructed a Lindelöf space $X$ such that $\operatorname{dim} X=0$ and $X^{2}$ is normal but has $\operatorname{dim} X^{2}>0$.

The aim of this paper is to prove that no product of subspaces of Sorgenfrey lines can serve as a counterexample to the product conjecture. Another aim is to give a full answer to one of the questions raised by Mrowka [Mr2] in the conference of 1972 which says: "We still do not know if subspaces of $S^{n}\left(n=2,3, \ldots, \aleph_{0}\right)$ are strongly 0-dimensional."

The familiar Sorgenfrey space $S$ is defined to be the space of real numbers with the class of all half-open intervals $[a, b), a<b$, as a base. It is a well-known fact that $S$ is Lindelöf, first countable, $N$-compact and also has $\operatorname{dim} S=0$.

A Tychonoff space $X$ is called strongly zero-dimensional provided that $\operatorname{dim} X=0$.

The following theorem (see e.g., $[\mathbf{G}]$ ) characterizes the class of all strongly zero-dimensional spaces.

1.1 THEOREM. For a Tychonoff space $X$, the following conditions are equivalent:

Received by the editors August 9, 1976 and, in revised form, October 19, 1977.

AMS (MOS) subject classifications (1970). Primary 54F45; Secondary 54F50.

Key words and phrases. Completely regular, cozero cover, cozero set, $N$-compact, order of a cover, Tychonoff.

'The results in this paper are contained in the author's doctoral dissertation, written at SUNY at Buffalo under the direction of Stanislaw Mrowka in February 1976.

- American Mathematical Society 1978 
(a) $X$ is strongly zero-dimensional.

(b) $\beta X$ is strongly zero-dimensional.

(c) Every cozero-set of $X$ is a countable union of clopen sets of $X$.

It can be easily seen now that a Lindelöf space which has a base consisting of clopen sets must be strongly zero-dimensional. Since $S^{2}$ fails to be Lindelöf, there is no easy way to determine $\operatorname{dim} S^{2}$. The fact that $\operatorname{dim} S^{n}=0$ for all $n$ was proved only in 1972 [Mr1], [Te1]. Prior to that, several researchers have proved that $\operatorname{dim} S^{2}=0$ [Nyikos, Fund. Math. 79 (1973), 131-139], but their arguments could not be generalized, even to $S^{3}$. An interesting parallel is that Terasawa (private communication) has shown that $S^{2}$ is hereditarily strongly zero-dimensional; his proof cannot be generalized even to $S^{3}$.

2. The covering dimension of subspaces of product of Sorgenfrey lines. It is the time now to discuss the main result of this paper.

2.1 Proposition. Let $Y$ be any strongly zero-dimensional metrizable space and $X \neq \varnothing$ be a subspace of $S^{n} \times Y$ (where $n$ is a fixed integer). Then $X$ is strongly zero-dimensional.

Proof. Let $X$ be a nonempty subspace of $S^{n} \times Y$. By Theorem 1.1, it is sufficient to prove that each cozero-set of $X$ is the union of countably many clopen sets in $X$.

The proof will be carried out in several steps (I-II).

I. For any $z=\left(z_{1}, \ldots, z_{n}\right) \in S^{n}$, define

$$
V_{i}(z)=\left[z_{1}, z_{1}+1 / i\right) \times \cdots \times\left[z_{n}, z_{n}+1 / i\right) .
$$

For each integer $i \geqslant 1$, choose the sequence $\left\{u_{i k}\right\}$ in $S^{n}$ such that $S^{n}=$ $\cup_{k=1}^{\infty} V_{i}\left(u_{i k}\right)$. For simplicity, we let $V(i, k)=V_{i}\left(u_{i k}\right)$, and, for $z=$ $\left(z_{1}, \ldots, z_{r}\right), A \subset S^{n}, B \subset Y$, we let $A \cap_{\times} B=(A \times B) \cap X, z(k)=z_{k}$ $(k<r)$.

Since $\operatorname{dim} Y=0$, therefore $Y$ has a basis $\mathscr{B}=\cup_{i=1}^{\infty} \mathscr{B}_{i}$ consisting of clopen sets, where each $\mathscr{B}_{i}$ is a locally finite family (see [E, p. 291]).

II. Let $U$ be any cozero set of $X$ determined by a continuous function $f$ : $X \rightarrow[0,1]$ in such a way that $U=\bar{f}^{1}(0,1]$. For natural numbers $i, k, l$, and for each $G \in \mathscr{B}_{i}$, define

$$
\begin{aligned}
W_{G}(i, k, l)=\left\{x=(z, y) \in X \mid f\left(V_{i}(z) \cap_{\times} G(y)\right) \subset(1 / l, 1],\right. \\
\text { where } \left.z=\left(z_{1}, \ldots, z_{n}\right) \in S^{n}, y \in Y \text { and } G(y)=G\right\} \\
\cap V(i, k) \cap_{\times} G ; \\
O_{G}(i, k, l)=\cup\left\{V_{i}(z) \cap_{\times} G(y) \mid(z, y) \in W_{G}(i, k, l)\right\} \\
\cap V(i, k) \cap_{\times} G .
\end{aligned}
$$

(3) If $\left(z_{1}, y_{1}\right) \in O_{G}(i, k, l)$ and $\left(z_{2}, y_{2}\right) \in V(i, k) \cap_{\times} G$ such that $z_{2}(j)>$ 
$z_{1}(j)(j=1, \ldots, n)$, then $\left(z_{2}, y_{2}\right) \in O_{G}(i, k, l)$.

Define

$$
\hat{O}_{G}(i, k, l)=\mathrm{cl}_{X} O_{G}(i, k, l) \backslash O_{G}(i, k, l) .
$$

One can notice that $\hat{O}_{G}(i, k, l) \subset V(i, k) \cap_{\times} G$, and that the closure of $O_{G}(i, k, l)$ is the same as its Euclidean closure in $V(i, k) \cap_{\times} G$.

One can also notice that

(5) if $\left(z_{1}, y_{1}\right) \in \hat{O}_{G}(i, k, l)$ and $\left(z_{2}, y_{2}\right) \in V(i, k) \cap_{\times} G$ such that $z_{2}(j)>$ $z_{1}(j)(j=1, \ldots, n)$, then $\left(z_{2}, y_{2}\right) \in \mathrm{cl}_{X} O_{G}(i, k, l)$.

Define:

(6) $T_{G}(i, k, l)=\cup\left\{N(x): x \in \hat{O}_{G}(i, k, l)\right.$ and $f(N(x)) \subset(1 /(l+1), 1]$, where $N(x)$ is a basic neighborhood at the point $x\} \cap V(i, k) \cap_{\times} G$;

$$
F_{G}(i, k, l)=\operatorname{cl}_{X} O_{G}(i, k, l) \cup T_{G}(i, k, l) \subset V(i, k) \cap_{\times} G .
$$

Then $F_{G}(i, k, l)$ is a clopen subset of $X$ (see Observation 1 below).

Define

$$
F(i, k, l)=\cup\left\{F_{G}(i, k, l) \mid G \in \mathscr{B}_{i}\right\} .
$$

Then $F(i, k, l)$ is clopen in $X$ because $\mathscr{B}_{i}$ is locally finite.

We can easily prove that $U=\cup\{F(i, k, l) \mid i, k, l\}$ (see Observation 2).

Observation 1. $F_{G}(i, k, l)$ is a clopen subset of $X$.

It suffices to prove that $F_{G}(i, k, l)$ is closed since it is clearly open. To show that $F_{G}(i, k, l)$ is closed, it suffices to prove that $\operatorname{cl}_{X} T_{G}(i, k, l) \backslash T_{G}(i, k, l)$ $\subset F_{G}(i, k, l)$. Let $(z, y) \in \mathrm{cl}_{X} T_{G} \backslash T_{G}$. If $(z, y) \notin \mathrm{cl}_{X} O_{G}(i, k, l)$, there exists a basic neighborhood $N_{1}$ at $(z, y)$ such that $(z, y) \in N_{1} \subset V(i, k) \cap_{\times} G$ and $N_{1} \cap O_{G}(i, k, l)=\varnothing$. Since $(z, y) \in \mathrm{cl}_{X} T_{G} \backslash T_{G}$, there exists a point $\left(z_{1}, y_{1}\right)$ $\in N_{1} \cap T_{G}$. From (6), we can find $\left(z_{2}, y_{2}\right) \in \hat{O}_{G}(i, k, l)$ such that $\left(z_{1}, y_{1}\right) \in$ $N\left(\left(z_{2}, y_{2}\right)\right)$. Using (5), we get $\left(z_{1}, y_{1}\right) \in \mathrm{cl}_{X} O_{G}(i, k, l)$. Therefore $N_{1} \cap$ $O_{G}(i, k, l) \neq \varnothing$, which is a contradiction. Therefore $(z, y) \in \operatorname{cl}_{X} O_{G}(i, k, l)$ and consequently $(z, y) \in F_{G}(i, k, l)$.

Observation 2. $U=\cup\{F(i, k, l) \mid i, k, l\}$.

Let $(z, y) \in U \cap S^{n} \times Y$. By continuity of $f$, there exist $l_{0}, i_{0} \geqslant 1$ and $G \in \mathscr{B}_{i_{0}}$ such that $f\left(V_{i_{0}}(z) \cap_{\times} G(y)\right) \subset\left(1 / l_{0}, 1\right]$, where $G(y)=G$. Let $k_{0} \geqslant$ 1 be such that $z \in V\left(i_{0}, k_{0}\right)$. Then $(z, y) \in F_{G}\left(i_{0}, k_{0}, l_{0}\right)$ which completes the proposition. We can proceed now to our main theorem.

2.2 TheOREM. Let $Y$ be any strongly zero-dimensional metrizable space and $X \neq \varnothing$ be a subspace of $S^{\aleph_{0}} \times Y$. Then $X$ is strongly zero-dimensional.

Proof. Let $U$ be any cozero set of $X$ determined by a continuous function $f: X \rightarrow[0,1]$ in such a way that $U=f^{-1}(0,1]$. Then $U=\bigcup_{j=1}^{\infty} U_{j}$, where $U_{j}=\left\{x=\left(x_{1}, x_{2}, \ldots, y\right) \in U: f\left(N_{1} \times \cdots \times N_{j} \times S^{\aleph_{0}} \times G(y) \cap X\right) \subset\right.$ $(0,1]$, where $N_{1} \times \cdots \times N_{j} \times S^{\kappa_{0}} \times G(y) \cap X$ is some basic neighborhood at the point $x$ \} for $j=1,2, \ldots$. For each natural number $j \geqslant 1$, write $U_{j}$ as a countable union of clopen sets (use the same construction as in 
Proposition 2.1). It is clear that $U=\cup_{j=1}^{\infty} U_{j}$ can be written as a countable union of clopen sets which completes the proof of the theorem.

We shall list various corollaries to the above theorem.

2.3 COROLlARY. The space $S^{m} \times Y$ is hereditarily strongly zero-dimensional for every strongly zero-dimensional metrizable $Y$ and all $1 \leqslant m \leqslant \aleph_{0}$.

2.4 Corollary. If $X \neq \varnothing$ is a separable subspace of $S^{\kappa_{0}} \times Y$, then $X^{m}$ is strongly zero-dimensional for all cardinals $m$.

Proof. Let $\Lambda: N \rightarrow N \times N$ be a fixed bijection. Define the map $\sim$ : $\left(S^{\kappa_{0}} \times Y\right)^{\kappa_{0}} \rightarrow S^{\kappa_{0}} \times Y^{\kappa_{0}}$ by the rule $\sim\left(x_{1}, x_{2}, \ldots\right)=$ $\left(x_{\Lambda 1}, x_{\Lambda 2}, \ldots, y_{1}, y_{2}, \ldots\right)$, where $x_{i}=\left(x_{1, i}, x_{2, i}, \ldots, y_{i}\right) \in S^{\kappa_{0}} \times Y$ for $i=$ $1,2, \ldots$ It is clear that $\sim$ is a homeomorphism from $\left(S^{\kappa_{0}} \times Y\right)^{\kappa_{0}}$ onto $S^{\kappa_{0}} \times Y^{\kappa_{0}}$.

Let $\sim\left(X^{\aleph_{0}}\right)=\tilde{X}$. Then $\tilde{X} \subset S^{\kappa_{0}} \times Y^{\aleph_{0}}$ is strongly zero-dimensional and hence $X^{\kappa_{0}}$ is also strongly zero-dimensional.

Now, let $m$ be any cardinal $\geqslant \kappa_{0}$, and let $U$ be any cozero set of $X^{m}$ which is determined by a continuous map $f: X^{m} \rightarrow[0,1]$ in such a way that $U=f^{-1}(0,1]$. By the Gleason Theorem (see [I]), we get the existence of continuous maps $g$ and $\pi$ such that the following diagram commutes.

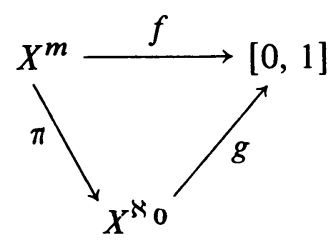

It is clear that $U=\pi^{-1}\left(g^{-1}(0,1]\right)$ is a countable union of clopen sets in $X^{m}$.

2.5 COROllaRy. If $\varnothing \neq X \subset S$, then $X^{m}$ is strongly zero-dimensional for any cardinal $m$.

The proof follows immediately from Corollary 2.4 and the fact that $S$ is a hereditarily separable space.

3. Significance of the main results. As we explained, our results were prompted by the product conjecture "The product of any two strongly zero-dimensional spaces is still strongly zero-dimensional." However, these results are also relevant for other problems, e.g. for the problem of hereditary strong zero-dimensionality of various product spaces, strong zerodimensionality of $N$-compact spaces and also for the following:

The Union Problem $(U)$. If $X_{1}$ and $X_{2}$ are disjoint strongly zero-dimensional subspaces of $X$, with $X_{1}$ closed in $X$, and $X=X_{1} \cup X_{2}$, then is $X$ also strongly zero-dimensional? 
We wish to discuss our results in view of the above problems. It has been recently demonstrated that $N$-compact spaces need not be strongly zerodimensional. The first example was given in 1972 by Mrowka [Mr2], and another one, still unpublished, by E. Pol and R. Pol. Both of these examples are quite complex; it is therefore reasonable to inquire whether some wellknown space can serve as an example.

As we mentioned before, the product conjecture has been solved negatively by Wage [W] and Przymusiński [P]. The Union Problem also has been solved negatively by Terasawa [Te2].

It is easy to see that $S^{\varkappa_{0}}$ is hereditarily $N$-compact. Consequently, for some time, it was conjectured that subspaces of $S^{\kappa_{0}}$ could provide an example of $N$-compact nonstrongly zero-dimensional space. Our results eliminate this possibility; more exactly, they eliminate the following spaces from these considerations:

(a) subspaces of $S^{\aleph_{0}}$,

(b) products of subspaces of $S$.

Observe also that, for large $n, S^{n}$ is not even closed hereditary strongly zero-dimensional. Indeed, if we take the nonstrongly zero-dimensional $N$ compact space $\mu$ (see [Mr2]), then, for large $n$, we have $\mu \subset_{\mathrm{cl}} N^{n} \subset_{\mathrm{cl}} S^{n}$.

Moreover, our results may be considered as generalizations to those found in [Mr1] and [Te1].

To conclude this section we will comment on the connection of our results with the so-called intermediate topology (described in [Ta]). These matters are related to both the Product and the Union Problems. In this matter, one considers a space $X$ with a distinguished subset $M$ such that $M$ and $X \backslash M$ are both metrizable strongly zero-dimensional spaces; the theorem in [Ta] asserts that, under a certain additional assumption, $X$ is strongly zero-dimensional.

On the other hand, by our results, $M \times S^{\kappa_{0}}$ and $(X \backslash M) \times S^{\kappa_{0}}$ are both strongly zero-dimensional. Thus, if $X \times S^{\kappa_{0}}$ (with an $X$ satisfying the assumption of the required theorem in [Ta]) would fail to be strongly zero-dimensional, this would provide a counterexample to the Product Problem as well as to the Union Problem.

\section{REFERENCES}

[E] R. Engelking, Outline of general topology, North-Holland, Amsterdam, 1968.

[G] L. Gillman and M. Jerison, Rings of continuous functions, Van Nostrand, New York, 1960.

[I] J. R. Isbell, Uniform spaces, Math. Surveys, American Mathematical Society, Providence, R.I., 1964.

[Mr1] S. Mrowka, Recent results on E-compact spaces and structures of continuous functions (Proc. Univ. of Oklahoma Topology Conf., 1972), Univ. of Oklahoma, Norman, 1972, pp. 168-221.

[Mr2] Recent results on E-compact spaces, TOPO-General Topology and its 
Applications, Lecture Notes in Math., vol. 378, Springer, Berlin and New York, 1974, 298-301. [P] T. C. Przymusiński, On the dimension of product spaces and an example of $M$. Wage (to appear).

[Ta] H. P. Tan, Doctoral Dissertation, Buffalo University, 1973.

[Te1] J. Terasawa, On the zero-dimensionality of some nonnormal product spaces, Sci. Rep. Tokyo Kyoiku Daigaku 11 (1972), 167-174.

[Te2] __, NUR and their dimensions (to appear).

[W] M. Wage, The dimension of product spaces (to appear).

DEPARTMENT OF MATHEMatics, Yarmoux UnIVERSTTY, IRBID, JoRDAN 